\title{
To What Extent Does Fandom Wikia Act as a Model for Labor Exploitation?
}

\author{
Justin Lim ${ }^{1}$ \\ ${ }^{1}$ Long Beach Polytechnic High School, Long Beach, CA, USA
}

\section{ABSTRACT}

Founded in 2004, the company, Fandom, provided a new outlet for fans to engage with the media they love: a platform to edit and view information about their interests, as well as meet others who share those interests. It is possible, however, that Fandom has taken advantage of this as a vast pool of labor. As Fandom makes money from ad revenue and their users continue to make pages of information making ads more accessible, it comes into question whether or not this is a model for labor exploitation. In order to classify it as such, two conditions needed to be fulfilled to qualify Fandom as a model: is Fandom monopolistic and does it take advantage of its users. Whereas the monopolistic aspect can be determined by seeing if other media fill the niche Fandom does, the second aspect will be determined using Katz's Uses and Gratifications Theory. A survey was conducted from user responses to analyze how users view Fandom. It was determined from the 50 responses that Fandom fits the model as it is the only media platform that fulfills an informational and social niche concurrently. The website also fulfills all aspects of Katz's Theory, therefore, the second condition for labor exploitation is fulfilled. Although Fandom acts as a model for labor exploitation, this does not outline the moral aspects of its activities; this only classifies it as a model to be utilized for reference as technology continues to make controversial issues such as labor exploitation more ambiguous.

\section{Literature Review}

In the past two decades alone, the Harry Potter fandom has been a forefront example of an exponentially growing fandom participatory culture. What was at first a seven-book series has now expanded into a movie franchise, its own section in Universal Studios, and has been moved into spin-off content as well, all of which have been incredibly profitable. Aside from the lucrative marketing of the Harry Potter franchise, the fandom was birthed during the rise of technology where many outlets for creativity were opened to fans, and as a result, it paved the way for fandom content creation today. This engagement with participatory culture translates to this era in which technology is often sourced as a medium, not only for content creation but also connecting with other people to share and appreciate said created content in the process. However, with this sudden surge comes its downfall as corporations have taken advantage of this inherent creative quality in fanbases and have exploited it for their own financial self-interest. One of the primary culprits amongst the many methods that corporations utilize has been wikis: websites that provide information that has been created and edited through the collaborative efforts of multiple users across the site. Such websites have been at the forefront of using this source of free fan labor, however, is it enough to qualify it as a legitimate case for labor exploitation? To better understand this phenomenon that ties corporate profit with content creation, an understanding of audience motivations behind content creation should be established before diving into further analysis regarding labor exploitation with Fandom Wikia, a primary instigator within this controversy, being used as a model of research 


\section{Audience Motivation Through Uses and Gratifications Theory}

Audience behavior is a significant topic of interest within this study, as gathering an understanding of what motivates an audience to engage with media and merchandise is essential to knowing what these companies take advantage of when they exploit fan labor. One of the primary theorems used to understand this is the Uses and Gratifications Theory, most commonly associated with American sociologist Elihu Katz. Uses and Gratifications Theory is a commonly known model within Communications Studies of which its primary purpose is to identify motivations within the reader to engage with media and/or merchandise. This theory is broken down into five motivations best broken down in the Uses and Gratifications Research by Katz as well as University of Maryland professors, Jay Blumler and Michael Gurevitch. According to Blumler, Gurevitch and Katz's piece, Uses and Gratifications Theory is a theory that analyzes the purposes behind audience behaviors and breaks down into five motivations for maintained, engaged audience behavior: escapism, education, experience, identity, and relationship. "Escapism" acts as one of the typical purposes that entices viewers to start their engagement with a movie or a television show because it functions as the "easement via mass media consumption" or an escape from everyday life; this could range from a comedic movie or an engaging video game. "Education" allows viewers to enrich themselves through a continual release of content that either teaches academic values, moral values, or just keeping up with the media itself in the process of enjoying content; informational television or podcasts act as a simple form of educational engagement. "Experience", as a purpose for viewers to engage with media, functions as a way to "satisfy certain needs to make up for "impoverished life opportunities," essentially making their engagement contingent upon the uniqueness of the content they watch; from a romantic movie to a television show that tours different parts of the world, the content of the show usually targets what a specific audience may be deprived of (Katz et al, 1973).

Whereas the above purposes focus on an introduction to a media-related needs and small factors that keep those individuals engaged, the latter two purposes introduced by Uses and Gratifications Theory focus more heavily on what keeps these individuals committed. As detailed by University of Texas Associate Professor of Communications, Thomas Ruggiero, within the modern age, the new forms of mass communication media have resulted in "interpersonal and qualitative aspects of mediated communication" that contribute heavily to these latter two aspects of Uses and Gratifications (Ruggiero, 2000). "Identity", as a gratifying purpose, allows for the validation of particular values through the "consumption of congruent media materials," and creates a stronger relationship for the viewer every time they choose to re-engage with the material they enjoy. "Relationship" has developed more as a purpose in the modern day as the rise of technology has allowed "valued social groupings" to be able to connect with one another and establish connections (Katz et al, 1973). This distinct aspect that technology has been able to engender gives groups a sense of belonging as being able to connect with other people who can relate and enjoy the same content reinforces the motivation to keep up with these media-related needs. These five purposes stand as the main motivations behind media engagement and will help to better understand how Fandom utilizes these motivations to help garner revenue.

\section{Defining Labor Exploitation}

With this understanding of Uses and Gratifications Theory in mind, the other component of this analysis is to outline what qualifies as labor exploitation. The Stanford Encyclopedia of Philosophy states that exploitation is defined as using "another person's vulnerability for one's own benefit" (Zwolinski et al, 2001). The connotations of the wording may imply that this type of behavior is immoral, however, it's not necessarily wrong all time, but rather different from case to case. For example, a chess player is not condemned for exploiting a weakness in his opponent's strategy. Therefore, the general idea of exploitation is defined by whether or not a vulnerability is being used for the benefit of an entity and not the moral implications surrounding it.

However, this only limits the definition to the general concept of exploitation. Two additional conditions limit the model to labor exploitation. In his analysis commenting on measurements of labor exploitation moving into 
the modern age, University of North Texas Associate Professor of Sociology, Milan Zafirovski, measured labor exploitation models over time through the Marxist and Neoclassical lenses and one component of note that was a part of Zafirovski's analysis was "monopoly power" (Zafirovski, 1999). Although other conditions were put into place such as the imbalance of the profits to capital ratio, this is not as applicable to the study as Fandom has access to a large pool of labor without paying these particular users. However, the monopolistic aspect fits within this exploitative model as companies in the future take over a particular commodity, they can exploit the consumers of a particular commodity with no room for escape with all revenue directed towards them. Thus, both aspects, utilization of vulnerabilities and monopolistic control, are the main factors in determining modern forms of labor exploitation.

\section{Fandom Wikia, Fan Gratification, and Fan Labor}

With this understanding of Uses and Gratifications Theory and labor exploitation in mind, identifying how this ties into fandom content creation is quite simple. There are three levels of engagement with a fandom, each involving more activity than the last. For members of the fandom, there are those who watch the content itself to fulfill their respective gratifying purposes, a "passive fan"; "active fans," who will purchase merchandise related to their fandom; and the small percentage of "content creators," devoted towards content creation which in his Critical Studies in Mass Communication, University of Southern California Communications Professor, Henry Jenkins, attributes to the need for "appropriating or reappropriating" content in whatever outlet the particular creator chooses ranging from fan art to fan fiction (Jenkins, 1988). Additionally, as explained above through Uses and Gratifications Theory, technology has been able to further reinforce these relationship needs by allowing those with similar interests to connect and interact, and with the introduction to content creation, it has also allowed said content to be shared across the internet. This has formulated communities of support for one another and as much as it gratifies a relationship need, it also encourages fans to continue to maintain their engagement, even after their particular franchise may have ended its tenure.

In order to apply this better within the realm of wikis, one of the largest wiki-based sites will be used as a model to study: Fandom Wikia. Founded in 2004, Fandom Wikia has been a leading example of exploitative digital labor in the modern age, but exactly how does it do so? The company's website itself functions quite similarly to Wikipedia in that it is a source of information included with pages upon pages of information regarding different pop culture, such as protagonists from film or television, details about the plotlines of specific episodes, or running gags within shows; this is further applicable within video games with exact details of characters or gameplay. Anybody is able to access the content, making it both fan-researched and community-researched, and while this open editing may not allow for any officially certified credentials, passionate and committed fans typically produce the most accurate information that stays true to the shows that they love. As long as the user makes an account they are able to participate within the contribution process. If these users would like a better understanding of the website's functionality, they are also given an opportunity by Fandom to further understand it through the eponymous "Fandom University" which "provides instructions, help, and advice to users at every level of experience" in order to better educate them and maximize their experience (Fandom, 2016). Furthermore, users are permitted access to forums and discussion groups that allow them to further analyze, theorize, or discuss aspects of the show or issues within the wiki that require it, which provides users a more interactive experience between fellow fans within the community, and motivates them to commit more to the media they enjoy. With such a unique concept of a website that offers so much to do, Fandom has gathered a community of around 23 million users within the community, and that does not include the many web surfers who may not have accounts but are still free to utilize the website (Fandom, 2020).

Although the engagement with other fans is a definite social perk integrated into the website, the key to Fandom's lucrative business is the very concept of content creation. The essential relationship allows users to help educate fans who might be introducing themselves to a new media, and this relationship alone encourages the user to further engage themselves with the content. Beyond this strict relationship, however, "each wiki page has associated with it a discussion tab where users discuss the page's contents," allowing further interaction between users to enjoy 
and enrich themselves (Mittell, 2009). Furthermore, users are permitted access to forums and discussion groups that allow them to further analyze, theorize, or discuss aspects of the show or issues within the wiki that require it, which provides users a more interactive experience between fellow fans within the community, and motivates them to commit more to the media they enjoy. This dynamic in which users can share, interact, and educate one another over similar interests, offers a sense of belonging and understanding between one another sets apart Fandom Wikia. Not only have wikis within this era become "a viable and usable knowledge management tool due to its ease of use and collaborative nature," but this outlet reinforces user relationship and interaction through the form of community that this website is able to construct (Pei Lyn Grace, 2009). Despite this range of benefits to help gratify the user, it is important to remember that the company has essentially opened itself to a large group of free labor, which therefore raises a question: how does Fandom Wikia turn a profit in the first place?

The company itself receives revenue through advertisements on its website. Additionally, according to "The Daily You" by University of Pennsylvania Communication Professor, Joseph Turow, he further clarifies that the main source of revenue with websites like these is through clicks. These clicks can only be received provided that there is a website page to access. Likewise, Fandom has access to a pool of fan labor that creates a myriad of website pages almost every day, which increases the chance of the Fandom website to show up "in [somebody's] search engine results and give [them] additional reasons to stay." As a result, the website "will have more opportunity to learn about [their users] and serve them ads," a key role to play so the company can earn revenue (Turow, 2011). What makes this digital labor more lucrative for Fandom Wikia is that the multitude of pages and articles that are created at such a rapid pace by their online users, comes to almost no cost to Fandom Wikia. This utilization of audience labor and their gratifications allows for a seemingly unfair control of the online advertising market, and with the consolidation of digital fan labor, a wide audience from a multitude of fandoms, and targeted advertising to each user, Fandom creates an extremely profitable dynamic for the company with virtually no labor cost. This does not mean there are no costs to the business that Fandom Wikia runs at all; there are still payments to managing officers of the business and maintenance of the website domain. Regardless of these costs to the business, their access to free labor allows these relatively minimal costs to be dismissed because they are outweighed by the abundant benefits provided by the community.

\section{Method}

\section{Purpose}

The purpose of conducting a survey as a means to analyze the exploitative dynamic following Fandom Wikia is to see the motives behind the contribution and putting it in line with the motivations set by Katz's Uses and Gratifications Theory. This is in no way intended to demean Fandom Wikia, but it is rather setting a precedent for what labor exploitation looks like within the modern technological age and if Fandom Wikia fits the definition for exploitation and how through the lens of Katz's Uses and Gratifications Theory and the monopolistic condition of Zafirovski's modern labor exploitation analysis. The survey is the best way to get accounts from a wide variety of users as there are millions on the site ranging from different backgrounds. Their experiences are vital to see the motivations behind the labor itself and if all aspects of both theories are fulfilled and utilized within the website.

\section{Techniques/Methods}

Although the dynamic in which Fandom Wikia is organized lets it act as a model for the possibility of modern labor exploitation, in order to reinforce this concept, a survey will be incorporated into the research to bridge this gap between hypothesis and validation. In order to achieve this, 50 users of the site will be surveyed through the Google Forms medium analyzing their site activity. 
The form will be posted on the forums of different subsections of the sites (i.e. Harry Potter, Game of Thrones, World of Warcraft, etc.). As there are so many users of the site, there is no way to formally conduct a proper random distribution of the survey, the method will be openly conducted and will be open for any users on the website to click on the link, but the survey will be closed upon reaching 50 responses. Demographic questions were incorporated; however, the survey was conducted without any need for identifying information, and the individual information will remain confidential. The focus of the questions is to get a general idea of how users utilize the website, for how long they are typically on the site, and their own experiences with using the website.

\section{Reading, Contributing, and Discussing}

All users have access to the same form, however, the answer for one specific question determines where the form diverges: "What is your main usage of the site?" This question provides the options: reading articles, contributing to articles, or contributing to discussion groups. These responses essentially divide the survey participants into groups by their respective activities on the website. Following this division, the questions remain relatively the same with some questions tailored more to the specific role the responder has chosen such as estimated contribution times and estimated activity time on the site.

\section{Concluding Data}

As explained, there are two main components of focus within the data. The first is the user input regarding motivations. As stated in the purpose of this chosen method, the specific user input analyzes what brings the users to use the site in the first place. This is used in tandem with Katz's Uses and Gratifications Theory to see the correlation between labor and media interactions based on these motivations. Based on responses on user opinion of the website, if all aspects are fulfilled within Fandom Wikia, it is clear to what extent media motivations are a vulnerability and are exploited within Fandom Wikia.

The second component is the monopolistic aspect of labor exploitation. One of the main questions asked of all participants was whether or not they receive the same services from another website that they do from Fandom Wikia and if so, what websites would they be. These responses will determine how wide Fandom Wikia dominates and has taken over to make revenue off of its users.

\section{Results}

\section{Participant Demographic Data}

The survey was issued over the course of two weeks at which it had received 50 responses and was closed. The racial diversity of the survey participants are as follows: 64\% White, 18\% Asian/Pacific Islander, 4\% African American, 4\% Mixed, and $10 \%$ other. The distribution of the genders was skewed with $72 \%$ of the individuals being male, $20 \%$ being female, and the remaining $8 \%$ preferring not to say. Additionally, half of our participants came from the 10-20 year old range whereas the remaining participants were spread out amongst the remaining older age ranges (36\%).

\section{Survey Data}

The responses from these particular participants revealed that most of them are typically very active users with approximately $62 \%$ reporting daily usage of the website. These participants were then broken down into respective groups to define their particular activity on the website as indicated by Table 1 . 
Table 1. Reported Main Activity on Fandom Wikia.

\begin{tabular}{|ll|}
\hline Main Activity & Total Percentage \\
\hline Reading Articles & $6.0 \%$ \\
Contributing to Articles & $60.0 \%$ \\
Contributing to Forums & $34.0 \%$ \\
\hline
\end{tabular}

The survey then divided the participants into different groups based on their respective reported main activity on the website. Those who contributed to articles were set to one set of questions while those who read articles or contributed to forums were delegated to another group. Those who reported article contribution had the same questions as the other group, however, they received additional questions asking about details on their article contribution. For participants who contributed to articles, they were asked on average times of their article contribution and approximate article contribution overall. These article contributions may be limited to simple edits on a page after the release of a new episode or the creation of an entirely new page.

Table 2. Average Article Contribution Frequency

\begin{tabular}{|ll|}
\hline Frequency of Contribution & Total Percentage \\
\hline Not that Often & $3.3 \%$ \\
2-3 Times a Week & $16.7 \%$ \\
4-6 Times a Week & $16.7 \%$ \\
Everyday & $63.3 \%$ \\
\hline
\end{tabular}

Table 3. Average Contribution Time Per Session

\begin{tabular}{|ll|}
\hline Contribution Time & Total Percentage \\
\hline $0-30$ minutes & $16.7 \%$ \\
$30-60$ minutes & $66.7 \%$ \\
$60+$ & $16.7 \%$ \\
\hline
\end{tabular}

The participants revealed particularly high contribution frequencies as indicated by Table 2, with a majority of the users who reported contributing as their main activity to be nearly every day. Furthermore, it seems that these users typically spend between 30 to 60 minutes on article contribution per session that they access the site to contribute. Amongst all participants, they were asked how long they typically access the website in one session as well as how many articles they typically view during each period of access to further determine how their time was spent on the site in comparison to their respective main activity. 
Table 4. Session Duration vs Article Access

\begin{tabular}{|llllll|}
\hline Session Duration & \multicolumn{2}{l|}{ Article Access per Session } & & \\
\hline & $<5$ & $5-10$ & $10-20$ & $20+$ & Total \\
$0-20$ min & 8 & 10 & 4 & 0 & 22 \\
$20-40 \min$ & 0 & 4 & 3 & 2 & 9 \\
$40-60$ min & 0 & 1 & 1 & 2 & 4 \\
$60+$ & 0 & 4 & 2 & 9 & 15 \\
Total & 8 & 19 & 10 & 13 & 50 \\
\hline
\end{tabular}

Additionally, these users were asked whether or not they view any other websites to access the same kind of information that they do on Fandom Wikia. Of all that responded, only 14 reported that they do. They were also asked to list which websites they visit to access this particular information. Those that were listed included: Gamepedia, A Wiki of Ice and Fire, Wikipedia, Reddit, Serebii, Pluspedia, Biblioteka Ossus, Tokusatsu Network, and other main social media platforms such as Facebook and Twitter. All users were also asked if they contribute to any forums or discussion groups. This was mainly to apply to the groups that contributed to articles or read articles to see if they involved themselves in any other activities aside from their main reported one.

Table 5. Forum Usage Amongst Individuals Reading or Contributing to Articles

\begin{tabular}{|llll|}
\hline & Uses Forums & Doesn't Use Forums & Total \\
Contributing to Articles & 20 & 10 & 30 \\
Reading Articles & 6 & 11 & 17 \\
Total & 26 & 21 & 47 \\
\hline
\end{tabular}

Those contributing to articles seem to have a higher rate of participating in forums while individuals who read articles usually didn't. This skewness for individuals contributing to articles may be attributed to heavier involvement within a particular Fandom group whilst those who usually read articles may only use Fandom for information.

Users were then asked of their personal opinions on the website and about their favorite aspects of the website. This section was entirely optional, however, 33 reported certain aspects of the website that they enjoyed or disliked. Many replied with general aspects such as editing capabilities, information access, and the communication functions on the website. Others replied that the "sense of community" is what they enjoy the most and that for one particular individual, it has become a "personal home." Contrarily, one individual commented on wikia's form of expansion to maximize revenue as unnecessary as they feel it is "trying too hard to be the next social media site," while others commented on the toxic nature of some of the site's users. Overall, these responses seemed to indicate a general enjoyment of Fandom Wikia for the many functions it serves to gratify the user's needs, however, there are some aspects that users disapprove of, but the benefits certainly seem to outweigh the disadvantages for this website and has allowed it to grow into the status Fandom has achieved today. 


\section{Discussion/Analysis}

As discussed within the method, there are two things to focus on with the results of this study, the user's perspectives to see if Fandom exploits its users through the lens of Uses and Gratifications Theory and whether or not Fandom fits the monopolistic model within the modern age. Both of these components are analyzed separately but are vital to understanding Fandom's potential for modern labor exploitation and how this model can be portrayed on a broader perspective within the current technological age.

The first component of understanding how this website utilizes user motivations will be analyzed by comparing the survey participants' opinions of the website and how they align with the motivations set out in Katz's Uses and Gratifications Theory. With these comparisons in mind, we can see to what extent that Fandom Wikia manipulates the common fan.

As stated in the results, participants were asked to state their opinions on the site's functionality and their favorite aspect of the website to get user insight on what brings users to the website initially. A few participants said that the website was an "attention trap" and "addicting" which fulfills the "Escapism" aspect of Uses and Gratifications Theory, as it seems that these users view Fandom as an alluring activity to capture their attention. Others commented that they come to the website to "check articles to refresh [their] memory" on the particular media they follow, thus fulfilling the "Education" aspect. One especially commented on the Status Article Nominations functionality which is applicable in some fandom groups that allow users to nominate an article for awards and merits. Based on this functionality, it fulfills the "Experience" aspect as it not only allows users to contribute to a larger purpose, but it also issues merits upon the user. The output of these motivations is clearly seen as indicated in the results recorded in Table 2 and Table 3 where $63.3 \%$ of these users report to contribute to articles every day and another $33.4 \%$ contribute on quite a frequent basis. Additionally, many of these users spend more than 30 minutes in a given session to contribute to articles in their respective fandoms. Thus, in both the escapist and experiential aspects of Uses and Gratifications, users seem to dedicate a lot of time if they commit themselves to article contribution.

The social aspects of the website, such as the forums and discussion groups are an integral part of the "Identity" and "Relationship" aspects. As one participant reported, the website has become a "personal home" for them as they identify with other individuals within the fandom they are a part of. It has become a home for them with a community of people they relate to, which fulfills the respective "Identity" aspect. As many users also report that they enjoy "the community" and "the interaction" on Fandom Wikia, the "Relationship" aspect is therefore also fulfilled as users build relationships with each other by bonding over a particular media.

Thus, as all aspects are fulfilled through the lens of Uses and Gratifications Theory, Fandom Wikia takes advantage of every motivation behind the media consumption of users in order to gain mass revenue from a wide population with distinct reasons to engage with media. The socially integrative aspects of Identity and Relationship seem to be a larger focus of Fandom Wikia as it takes the informational aspect of the website and incorporates this social aspect alongside it to allow users to collaborate and lets the website distinguish itself from other mass media platforms. This builds into the monopolistic control aspect that helps build the definition of modern labor exploitation.

As participants were asked what other sources they use to access the same functions of Fandom Wikia, one of the more reported sources with the most similar functions to Fandom Wikia was Gamepedia, which happens to be owned by Fandom. Other reported sources were Facebook, Twitter, and Reddit. While these may add a communicative component, they do not provide detailed facts and information as Fandom does but rather focus more on the socially integrative aspects. The other reported sources were A Wiki of Ice and Fire, Wikipedia, Serebii, Pluspedia, Biblioteka Ossus, and Tokusatsu Network. With the exception of Tokusatsu Network, which acts more as a news outlet, these other reported sources' only purpose are for information referencing and open editing, which seems to touch more on escapist and informational motivations for media interaction. Fandom Wikia in this case dominates, not only because a large population of active users don't really engage with other websites for the same purposes that they do Fandom Wikia, but also in the case that they do, these websites don't have as many functions as Fandom Wikia seems to have. 
Fandom combines the informational aspects of many Fandoms and integrates a larger social component that allows users to collaborate to the extent they do and has allowed it to garner the popularity it has.

By finding a way to touch upon all aspects of Uses and Gratifications, Fandom Wikia has given itself access to a way to monopolize a market that no other company can touch. There are many pre-existing websites that have given themselves the purpose of contributing to either informational outreach or social connectivity, but Fandom Wikia has bridged these two aspects and spearheaded pop culture as the focus in order to reach a wide population.

Fandom Wikia has fulfilled what could be considered a definition for modern labor exploitation. The incalculable profit to capital ratio aside, the company has not only taken advantage of a wide variety of media engagement motivations to garner profit that qualifies it for exploitation, but it has also created an untouchable model that would be difficult to outcompete. It has dominated the particular service it provides and continues to attract wider populations by continuing to expand its functionalities. This data, although not representative of the entire user population, allows for insight into the model Fandom has constructed and greater focus on the users behind it within their interaction of the website.

\section{Limitations and Implications}

There are many formal limitations with the study itself that may not yield as accurate data as may be necessary. The first of these limitations is the focus of the study. With a wide variety of users on the website, it is difficult to contact each and every user individually. With certain information undisclosed such as the number of wikis or amount of users within a wiki, it is very difficult to conduct a random sample for the data. Thus, an open, voluntary survey is the best possible option provided by the limitations. The survey was posted on multiple forums on the website as this function is one of the main advertised aspects of the site. The survey was also posted on administrators for wikis because a title usually indicated persistent activity on the website. The issue this causes with the data, however, is that nonresponse bias is engendered by users who may have an account but are no longer active, but due to the inability to contact these users directly, the survey is the best option. This may be an advantage to shed more light on the activities of more active users of the website, however, this disregards activities for all users of the website as a whole.

Another limitation of note was drawn from one of the participants who reported that their behavior on the site "varies when a series is in hiatus," whether it be for reshooting or if the series itself is over. Likewise, contribution rates may vary as the activity of certain series are infrequent; however, the results displayed highlight the average levels of activity for the website from February 15, 2020 to February 17, 2020.

Furthermore, provided modern proper practices of safe internet behavior, a nonresponse bias continues to occur for users who generally do not want to click on an informal link for a study, and thus this specific population is not included in the results. Although the survey has the appropriate amount of responses having capped at 50, it is important to proceed with caution when viewing and discussing the utilized results as proper random sampling did not occur and thus can't reflect the behaviors for millions of users; however, this does not deny that the results given shed insight on the motivations behind contribution on Fandom Wikia.

\section{Conclusion}

The dynamic of fandom relationships has slowly evolved over the course of its growth in tandem with the rise of technology. Although this slow transformation over time has created outlets for creativity, it has also encouraged manipulation and exploitation of this particular group of peoples. As the study indicates, Fandom Wikia is considered a modern form of labor exploitation as not only does it fit the general definition by taking advantage of their user's own media related motivations for their own benefit, but it also takes over with economic domination. By fulfilling the monopolistic condition and virtually surpassing the profit to capital ratio, Fandom Wikia fits a model for what could be considered modern labor exploitation. 
This particular model for labor exploitation does not necessarily outline the moral definitions behind it. As mentioned within the Stanford Encyclopedia of Philosophy, the moral underlying of this form of labor exploitation is simply based on a case by case situation. The company may be manipulating media motivations outlined by Uses and Gratifications Theory, however, it is simply an inevitable form of media that has been capitalized on by a business. Additionally, the monopolistic aspect is difficult to combat as Fandom has distinguished its model and gathered such a wide community that competitors are unlikely to arise. Whether or not it is legally valid for companies to exploit innocent individuals like fans in such a manner is not the purpose of this paper, but it is rather to set a precedent for what exact conditions should fit for labor exploitation within a modern age in which questions like these are simply ambiguous. It builds a model for what this kind of modern labor exploitation may look like in the future and sets a comparative model to define alongside it. This is able to classify what defining labor exploitation should be like in future generations where growing advancements in technology make it more difficult to distinguish.

\section{References}

"Fandom University.” Community Central, community.fandom.com/wiki/Fandom_University.

Jenkins, H. (1988). Star Trek Rerun, Reread, Rewritten: Fan Writing As Textual Poaching. Critical Studies in Mass Communication 5:85-107.

Katz, E., Blumler, J. G., \& Gurevitch, M. (1973). Uses and Gratifications Research. The Public Opinion Quarterly37(4):509-523.

Pei Lyn Grace, T. (2009), "Wikis as a knowledge management tool", Journal of Knowledge Management, Vol. 13 No. 4, pp. 64-74. https://doi.org/10.1108/13673270910971833

Mittell, J. (2009), "Praxis." Transformative Works and Cultures, journal.transformativeworks.org/index.php/twc/article/download/118/117? inline=1?inline

Ruggiero, T. E. (2000). Uses and gratifications theory in the 21st century. Mass communication \& society, 3(1), 337.

Statistics. (n.d.). Retrieved May 25, 2020, from https://community.fandom.com/wiki/Special:Statistics

Turow, J. (2011). A New Advertising Food Chain. In Daily You, pp. 65-87. Yale University Press

Zafirovski, Milan. (1999, April 05). Measuring and Making Sense of Labor Exploitation in Contemporary Society: A Comparative Analysis. https://pdfs.semanticscholar.org/a1fe/eed5ec8f588335e9d0da1ddede52218c74e1.pdf

Zwolinski, Matt and Wertheimer, Alan, "Exploitation", The Stanford Encyclopedia of Philosophy (Summer 2017

Edition), Edward N. Zalta (ed.), URL $=<$ https://plato.stanford.edu/archives/sum2017/entries/exploitation/>. 